\title{
Safety and immunogenicity of novel $5 T 4$ viral vectored vaccination regimens in early stage prostate cancer: a phase I clinical trial
}

To cite: Cappuccini F, Bryant R, Pollock E, et al. Safety and immunogenicity of novel 5T4 viral vectored vaccination regimens in early stage prostate cancer: a phase I clinical trial. Journal for ImmunoTherapy of Cancer 2020;8:e000928. doi:10.1136/jitc-2020-000928

- Additional material is published online only. To view please visit the journal online (http://dx.doi.org/10.1136/jitc2020-000928).

AVSH and IR contributed equally.

Accepted 26 May 2020

Check for updates

(C) Author(s) (or their employer(s)) 2020. Re-use permitted under CC BY-NC. No commercial re-use. See rights and permissions. Published by BMJ.

For numbered affiliations see end of article.

Correspondence to

Dr Irina Redchenko;

irina.redchenko@ndm.ox.ac.uk

Federica Cappuccini, ${ }^{1}$ Richard Bryant, ${ }^{2,3}$ Emily Pollock, ${ }^{1}$ Lucy Carter, ${ }^{1}$ Clare Verrill, ${ }^{2,4}$ Julianne Hollidge, ${ }^{2}$ Ian Poulton, ${ }^{1}$ Megan Baker, ${ }^{1}$ Celia Mitton, ${ }^{1}$ Andrea Baines, ${ }^{1}$ Armin Meier, ${ }^{5}$ Guenter Schmidt, ${ }^{5}$ Richard Harrop, ${ }^{6}$ Andrew Protheroe, ${ }^{7}$ Ruth MacPherson, ${ }^{8}$ Steven Kennish, ${ }^{9}$ Susan Morgan, ${ }^{10}$ Selena Vigano, ${ }^{11}$ Pedro J Romero, ${ }^{11}$ Thomas Evans, ${ }^{12}$ James Catto, ${ }^{13}$ Freddie Hamdy, ${ }^{2,3}$ Adrian V S Hill, ${ }^{1}$ Irina Redchenko (i) ${ }^{1}$

\section{ABSTRACT}

Background Prostate cancer (PCa) has been under investigation as a target for antigen-specific immunotherapies in metastatic disease settings for the last two decades leading to a licensure of the first therapeutic cancer vaccine, Sipuleucel-T, in 2010. However, neither Sipuleucel-T nor other experimental PCa vaccines that emerged later induce strong T-cell immunity. Methods In this first-in-man study, VANCE, we evaluated a novel vaccination platform based on two replicationdeficient viruses, chimpanzee adenovirus (ChAd) and MVA (Modified Vaccinia Ankara), targeting the oncofetal selfantigen 5T4 in early stage PCa. Forty patients, either newly diagnosed with early-stage PCa and scheduled for radical prostatectomy or patients with stable disease on an active surveillance protocol, were recruited to the study to assess the vaccine safety and T-cell immunogenicity. Secondary and exploratory endpoints included immune infiltration into the prostate, prostate-specific antigen (PSA) change, and assessment of phenotype and functionality of antigenspecific T cells.

Results The vaccine had an excellent safety profile. Vaccination-induced 5T4-specific T-cell responses were measured in blood by ex vivo IFN- $\gamma$ ELISpot and were detected in the majority of patients with a mean level in responders of 198 spot-forming cells per million peripheral blood mononuclear cells. Flow cytometry analysis demonstrated the presence of both CD8+ and CD4+ polyfunctional 5T4-specific T cells in the circulation. 5T4reactive tumor-infiltrating lymphocytes were isolated from post-treatment prostate tissue. Some of the patients had a transient PSA rise 2-8 weeks following vaccination, possibly indicating an inflammatory response in the target organ.

Conclusions An excellent safety profile and T-cell responses elicited in the circulation and also detected in the prostate gland support the evaluation of the ChAd0x1MVA 5T4 vaccine in efficacy trials. It remains to be seen if this vaccination strategy generates immune responses of sufficient magnitude to mediate clinical efficacy and whether it can be effective in late-stage PCa settings, as a monotherapy in advanced disease or as part of multimodality PCa therapy. To address these questions, the phase I/II trial, ADVANCE, is currently recruiting patients with intermediate-risk $\mathrm{PCa}$, and patients with advanced metastatic castration-resistant $\mathrm{PCa}$, to receive this vaccine in combination with nivolumab.

Trial registration The trial was registered with the U.S. National Institutes of Health (NIH) Clinical Trials Registry ( ClinicalTrials.gov identifier NCT02390063).

\section{BACKGROUND}

Prostate cancer $(\mathrm{PCa})$ is the most common non-cutaneous malignancy in men and the second leading cause of male cancer-related death in the Western world. ${ }^{1}$ If the disease progresses to metastatic castration-resistant prostate cancer (mCRPC), the current treatment options are mainly palliative. Two nextgeneration hormonal agents, abiraterone and enzalutamide, have improved treatment of mCRPC; however, resistance to these drugs eventually ensues, with the disease becoming lethal. Cancer immunotherapy may be a viable option for treatment of patients with advanced stage PCa. To date, the only therapeutic cancer vaccine to be approved by the US Food and Drug Administration is Sipuleucel-T, and is indicated for patients with asymptomatic or minimally symptomatic mCRPC. ${ }^{2}$ This cell-based immunotherapy targeting the prostatic acid phosphatase (PAP) has shown modest, though statistically significant, efficacy in clinical trials, increasing overall survival in treated patients by 4 months. However, no effect on time to tumor progression compared with the placebo group has been observed and the induced T-cell responses appeared weak. ${ }^{3}$ 
Another vaccine, ProstVac, targeting prostate-specific antigen (PSA) and composed of two poxvirus vectors, demonstrated an 8.5-month increase in median overall survival compared with the control group in the phase II study in mCRPC. However, T-cell immune responses against the vaccine-encoded antigen were modest, ${ }^{4}$ and no clinical efficacy was observed in the ProstVac phase III trial. ${ }^{5}$

In the current study, we targeted the tumor antigen 5T4 using an immunization platform based on the ChAd prime and MVA boost. 5T4 is an oncofetal antigen, also known as trophoblast glycoprotein, typically expressed during embryonic development, while its expression is very limited in normal adult tissue. ${ }^{6}$ However, 5T4 was reported to be upregulated in a wide range of solid malignancies, including but not limited to colon, kidney, lung, breast, stomach, ovaries and prostate cancer, and its expression has been correlated with poor prognosis in multiple indications, ${ }^{7-11}$ making 5T4 a promising target for a cancer vaccine.

ChAd-MVA vaccination platform, developed in our laboratories over a decade ago, has been shown to be the most powerful approach for inducing polyfunctional protective T-cell responses against antigens from diverse human pathogens in clinical trials. ${ }^{12-18}$ Preclinical evaluation demonstrated tumor protective efficacy of this vaccination strategy in murine prostate cancer models, ${ }^{19} 20$ thus providing a rationale for a phase I clinical study. Here, we report the results of the first-in-human study VANCE, evaluating the safety of ChAdOx1-MVA 5T4 vaccine in early-stage PCa. The induction of antigenspecific immune responses in both the blood and the prostate gland, the effect of low-dose cyclophosphamide (CTX) preconditioning on the immune responses and changes in serum concentration of PSA are also reported. Vaccine efficacy assessments are effectively not possible in this trial design because the selected patient cohort either had a curative surgical treatment shortly after vaccination or a subsequent very low risk of recurrent disease that would require a long follow-up period beyond the duration of this trial in order to assess any clinical impact of vaccination.

\section{METHODS}

\section{Study design}

VANCE was a phase I randomized open-label study designed to assess the safety and immunogenicity of ChAdOx1-MVA 5T4 vaccine with and without low-dose CTX in low-risk and intermediate-risk localized PCa. Participants were enrolled at the Oxford University Hospitals NHS Foundation Trust and the Sheffield Teaching Hospitals NHS Foundation Trust, in the UK.

The sample size was chosen to allow an initial descriptive report of the safety and immunogenicity of the vaccine in a first-in-human trial.

Randomization was performed by an independent statistician at the Centre for Statistics in Medicine, Oxford
University. Allocation was performed using a balancedblock stratified randomization.

\section{Study population}

The target population for this study was men newly diagnosed with low-risk or intermediate-risk PCa who had chosen radical prostatectomy (RP) as their treatment option, and men with low-volume low-risk prostate cancer on an active surveillance (AS) program and stable for at least a year. Patients eligible for the study had histologically confirmed PCa, PSA $\leq 20 \mathrm{ng} / \mathrm{mL}$, Gleason score $\leq 7$, clinical stage disease $\leq \mathrm{T} 2 \mathrm{c}$ and no evidence of metastases.

Exclusion criteria included history of immunodeficiency disease, systemic immunosuppression, allergic response to previous vaccinia virus vaccinations, seropositivity for hepatitis B surface antigen, hepatitis $\mathrm{C}$ virus or HIV.

\section{Study products}

Design and construction of ChAdOx1.5T4 vaccine has been described previously. ${ }^{20} \mathrm{ChAdOx} 1.5 \mathrm{~T} 4$ was manufactured to clinical good manufacturing practice by the Clinical Biomanufacturing Facility (University of Oxford, Oxford, UK). The ChAdOx1.5T4 vaccine has been administered at the dose of $2.5 \times 10^{10}$ virus particles (vp). MVA.5T4 vaccine was manufactured and supplied for the trial by Oxford BioMedica Ltd (TroVax), and has been described previously. ${ }^{21}$ The MVA.5T4 vaccine has been administered at the dose of $2.0 \times 10^{9} \mathrm{TCID}_{50}$ (equivalent to $1.2 \times 10^{8}$ plaque-forming units $\left.(\mathrm{pfu})\right)$. Both vaccines were administered by intramuscular injection in the thigh.

CTX (manufactured by Baxter) was self-administered orally at $50 \mathrm{mg}$ twice a day for a 7-day cycle before each vaccination.

\section{Study procedures}

Following screening, patients in the surgical arms were randomly allocated to six groups. Participants in groups 1 and 2 received one ChAdOx1.5T4 immunization followed by two MVA.5T4 boosts 4 weeks apart and underwent RP at week 12 post-enrollment. Participants in groups 3 and 4 received three MVA.5T4 immunizations 4 weeks apart and also underwent RP at week 12 post-enrollment. This immunization schedule was termed a "standard" vaccination regimen. Participants in groups 5 and 6 were administered with ChAdOx1.5T4 vaccine followed by MVA.5T4 vaccination 1 week later and underwent RP at week 4 postenrollment. This immunization schedule was termed an "accelerated" vaccination regimen. Patients on the AS program were randomized to groups 7 and 8 and received an accelerated vaccination regimen followed by on-study prostate biopsy at week 10 post-enrollment. The participants in groups 2, 4, 6 and 8 received an oral course of low-dose CTX for 7 days prior to each immunization. The study treatment schedule is outlined in online supplementary figure S1.

Blood samples were drawn and clinical assessments conducted for safety, immunology and PSA monitoring 
prior to vaccination at day 0 and at each scheduled clinical time point following enrollment.

Fresh prostate tissue specimens were collected from surgical RP specimens and from on-study biopsies in AS patients for analysis of post-vaccination immune cell subsets within the prostate tissue following treatment.

\section{Endpoints}

Primary objectives of the study were safety of ChAdOx1-MVA 5T4 vaccination and the induction of immune responses to the vaccine-encoded tumorassociated antigen 5T4 in the blood following treatment. Secondary objectives of the study included comparative immunogenicity assessment of the standard and accelerated vaccination regimens, the effect of CTX preconditioning on the magnitude of immune responses, the effect of vaccination on the immune cells in the tumor and serum PSA change during the study.

\section{Ex vivo IFN- $\gamma$ ELISpot}

Ex vivo IFN- $\gamma$ ELISpot assay was performed at each scheduled time point in order to assess vaccine-induced immune responses. Briefly, $2.5 \times 10^{5}$ freshly isolated peripheral blood mononuclear cells (PBMCs) was plated in 96-well PVDF plates pre-coated with anti-IFN- $\gamma$ antibody and stimulated with eight individual pools each consisting of 10-12 15mer peptides overlapping by 10 amino acids, spanning the complete 5T4 protein sequence. Unstimulated cells were used as a background control and Staphylococcus enterotoxin B (SEB) and CMV/Epstein-Barr/Flu peptide mix (Mabtech $\mathrm{AB}$ ) were used as positive controls. After 18-24hours of incubation, plates were developed and the number of IFN- $\gamma$-producing cells (spot-forming cells, SFCs) was evaluated using an automated ELISpot reader (AID). 5T4-specific response was deemed to be positive at any time point when the mean number of SFCs per 1 million PBMCs was at least 40, and the number of spots per well at least two times the background. If a preexisting T-cell response was detected, a positive response due to vaccination was reported if the post immunization response was $\geq 2$-fold the response determined before immunization. Positive responses to individual pools were summed to give final SFC values per million PBMCs.

\section{In vitro culture of PBMCs and tumor-infiltrating lymphocytes (TILS)}

PBMCs and TILs were cultured in vitro to expand 5T4-specific T cells. Briefly, $3 \times 10^{6}$ freshly isolated PBMCs $/ \mathrm{mL}$ was plated in complete medium with or without total 5T4 peptide pool $(5 \mu \mathrm{g} / \mathrm{mL})$ in the presence of recombinant human IL-7 (rhIL-7) (25 ng/ $\mathrm{mL})$. Every 3-4 days, the medium was replaced and rhIL-2 $(100 \mathrm{U} / \mathrm{mL})$ added to the cultures. PBMCs were harvested after 2 weeks and incubated overnight at $37^{\circ} \mathrm{C}$ before further analysis. To obtain TIL cultures, prostate tissue specimens were cut into small pieces and cultured in the presence of rhIL-2 $(6000 \mathrm{U} / \mathrm{mL})$ as described previously. ${ }^{22}$ TIL medium was replaced every
2-3 days and the total 5T4 peptide pool was added when immune cell cultures entered an exponential growth phase. TILs were typically harvested 1 week post-5T4 stimulation for further analysis.

\section{Flow cytometric analysis}

In vitro cultured PBMCs and TILs were incubated with $1 \mu \mathrm{g} / \mathrm{mL}$ of co-stimulatory antibodies $\alpha \mathrm{CD} 28 / \alpha \mathrm{CD} 49 \mathrm{~d}$ (eBioscience) followed by stimulation in 96-well plates with complete medium, SEB $(1 \mu \mathrm{g} / \mathrm{mL})$ or total $5 \mathrm{~T} 4$ peptide pool $(5 \mu \mathrm{g} / \mathrm{mL})$. Following a 2-hour incubation, brefeldin $\mathrm{A}$ and monensin (BD Bioscience) were added and cells were incubated overnight at $37^{\circ} \mathrm{C}$. PBMCs were stained with surface antibodies, fixed/permeabilized and incubated with intracellular antibodies (online supplementary table S1). Dead cells were discriminated by live/ dead fixable staining (Life Technologies). Samples were acquired on an LSRII flow cytometer and analyzed with FlowJo software.

Patients were deemed to be "responders" when the percentages of IFN- $\gamma+$ TNF- $\alpha+C D 4+$ and CD $8+T$ cells after stimulation were higher than 0.02 and were at least 2-fold the background on analysis in at least one time point post-vaccination. In addition, for cultured PBMCs the delta values (5T4 value-background value) had to be $\geq 2$-fold the corresponding pre-vaccination baseline delta value.

\section{Tissue biopsy evaluation}

Archival diagnostic biopsies from AS patients collected within 1 year prior to enrollment, and on-study template or targeted biopsies obtained at week 10 post-enrollment, were evaluated. Tissue sections were stained immunohistochemically for expression of CD3 (clone LN10; Leica) and CD8 (clone C8/144B; Dako) at the ISO 15189 accredited NHS Cellular Pathology Laboratory at Oxford University Hospitals NHS Foundation Trust (Oxford, $\mathrm{UK})$. CD3+ and CD8+ T cells were enumerated according to the Tissue Phenomics approach employing a visual context random forest algorithm ${ }^{23}$ by Definiens $\mathrm{GmbH}$ (Munich, Germany). Epithelial and stromal regions were segmented via an in-house, pre-trained convolutional neural network ${ }^{24}$ and cell densities were calculated as the number of marker-positive cells per reference area $\left(\mathrm{mm}^{2}\right)$.

\section{Data presentation and statistical analysis}

Safety data are presented according to the frequency, severity and duration of solicited local and systemic reactogenicity signs and symptoms for 7 days following vaccination. Unsolicited adverse events (AEs) were recorded for 4 weeks post-vaccination. Statistical analysis of immunogenicity data was conducted using GraphPad Prism V.8. Comparisons between datasets were performed using non-parametric tests, as detailed in the figure legends. All $p$ values are two sided and considered statistically significant if $\mathrm{p}$ value $<0.05$. 


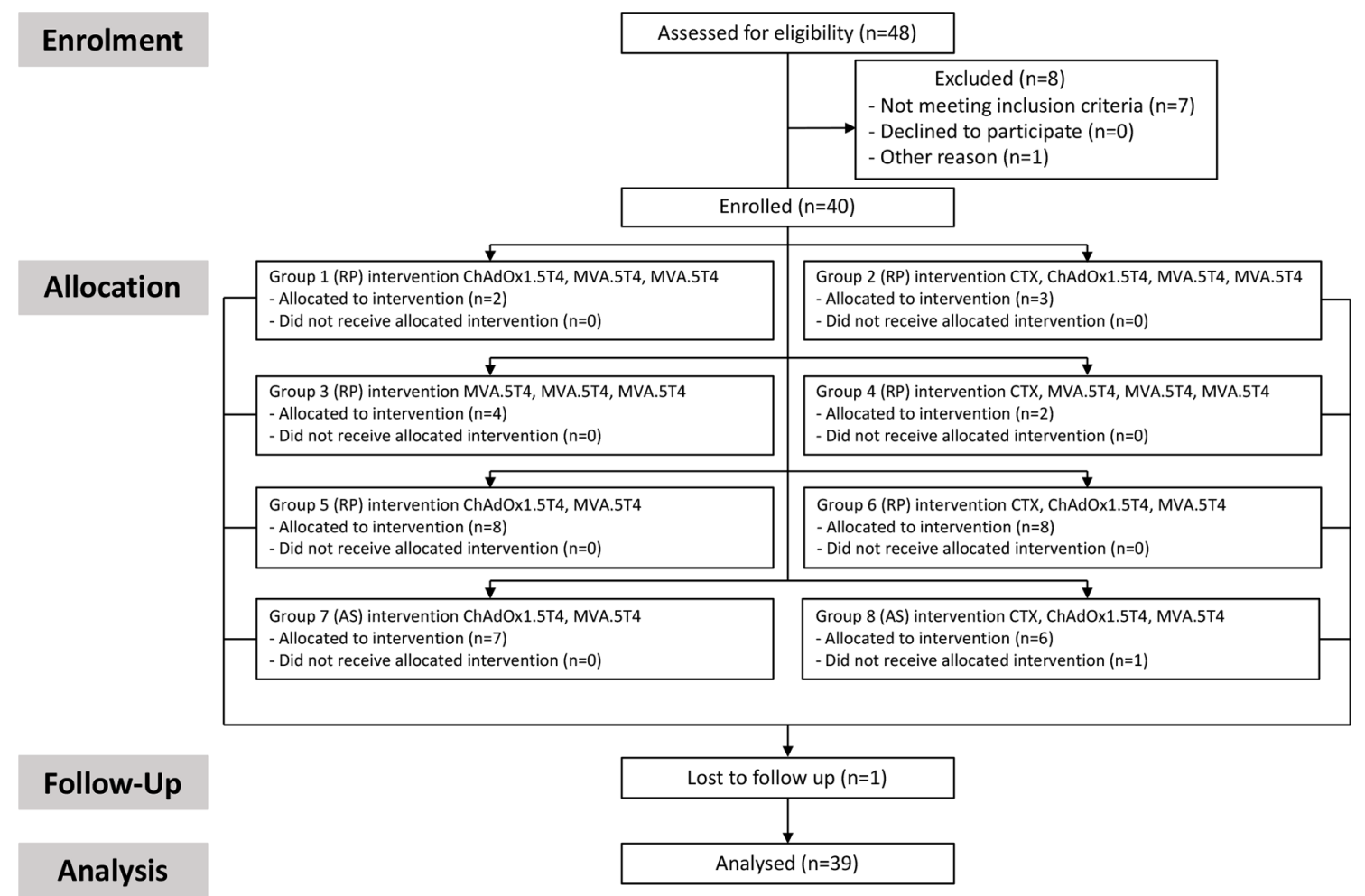

Figure 1 CONSORT flow diagram. Flow chart showing patients' participation in VANCE trial study from screening, enrollment, allocation, follow-up and analysis. AS, active surveillance; CTX, cyclophosphamide; RP, radical prostatectomy.

\section{RESULTS}

\section{Study participants and safety analysis}

Forty patients were enrolled into the VANCE randomized open-label phase I study between October 26, 2015 and October 19, 2017, with 39 participants completing the study treatment and eligible for analysis (figure 1). Median age, clinical stage and Gleason score of trial participants are presented in online supplementary table S2. VANCE clinical trial design is presented in online supplementary figure $\mathrm{S} 1$.

ChAdOx1.5T4 and MVA.5T4 vaccines were well tolerated. The majority of reported AEs related to vaccinations were mild in intensity (92\%) and were consistent with side effects observed for these vectors in other clinical trials. Mild to moderate pain at injection site was reported by $\sim 50 \%$ of patients. The majority of systemic AEs were graded as mild. Among the common systemic AEs usually seen post-replication-incompetent viral-vectored vaccines, feverishness, myalgia and fatigue were the most frequently reported, affecting $59 \%, 70 \%$ and $72 \%$ of individuals, respectively, and usually were resolved within 7 days post-vaccination.

There were no reported serious adverse events (SAEs) related to the vaccines. The two reported SAEs, hospital admissions due to an episode of hematemesis and an aortic surgery caused by ascending aortic aneurysm, were considered unrelated to the vaccines.

\section{Vaccine-induced T-cell responses in the blood}

Twenty-five of 39 (64\%) patients who completed the vaccination course and were eligible for analysis mounted
5T4-specific T-cell immune responses at one or more blood sampling timepoints, as measured by ex vivo IFN- $\gamma$ ELISpot assay, with a mean peak response of 198 SFCs per million PBMCs (figure 2A). The detected ex vivo 5T4-specific immune T-cell reactivity was attributed to de novo induced responses, and only 2 of 39 patients demonstrated pre-existing 5T4 responses, which increased after vaccination (figure 2A). The standard heterologous regimen (ChAdOx1-MVA-MVA 5T4, 4-week interval) induced an antigen-specific response in 4 of $5(80 \%)$ patients, and the standard homologous regimen (MVA-MVA-MVA 5T4, 4-week interval) in 4 of $6(66.7 \%)$ patients, with an overall immune response rate of $73 \%$. In the accelerated heterologous regimen (ChAdOx1-MVA 5T4, 1-week interval), 17 of $28(61 \%)$ patients showed cellular responses to $5 \mathrm{~T} 4$. The magnitude of antigen-specific immune response was not significantly different between standard homologous and heterologous vaccination regimens, perhaps due to the small number of patients in these arms (figure 2B). In other indications, the T-cell immunogenicity of ChAd-MVA regimens is consistently higher than that of homologous MVA regimens. ${ }^{25}{ }^{26}$ Also, comparable frequencies of antigen-specific $\mathrm{T}$ cells were observed following the standard heterologous and accelerated heterologous immunization schedules (figure 2B), as has been observed for other vaccinations such as Ebola. ${ }^{16}$ The CTX preconditioned arms did not show any increase in the magnitude of circulating T-cell responses compared with the vaccine-only arms (figure 2C). Importantly, immunizations against the 5T4 protein elicited T-cell 
A

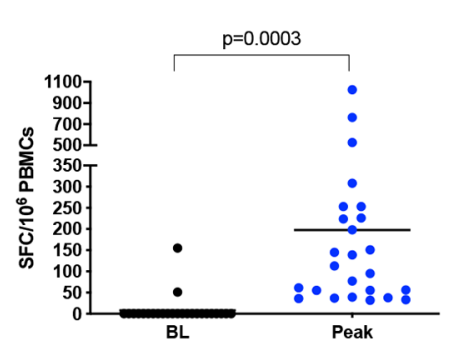

D

VAN-023 (RP)

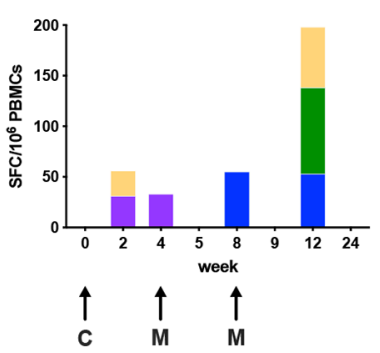

B

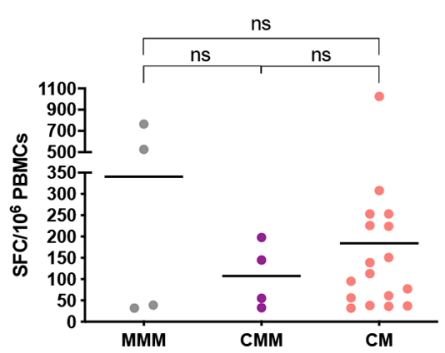

C

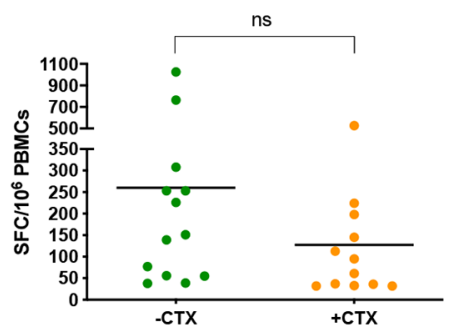

Figure 2 Ex vivo IFN- $\gamma$ ELISpot responses. Vaccine immunogenicity was assessed by ex vivo IFN- $\gamma$ ELISpot on freshly isolated peripheral blood mononuclear cells (PBMCs) stimulated with 8 pools of overlapping peptides covering the entire 5T4 protein sequence. Values of spot-forming cells (SFCs) per million PBMCs correspond to the sum of the responses to the single pools. (A) Dot plot representing peak responses for each individual patient compared with baseline (BL), pre-vaccination responses. Lines represent mean; the paired t-test $p$ value is shown. (B) Comparison of peak responses in patients receiving homologous standard vaccination (MVA-MVA-MVA 5T4: MMM), heterologous standard vaccination (ChAdOx1-MVA-MVA 5T4: CMM) and heterologous accelerated vaccination regimes (ChAdOx1-MVA 5T4: CM), irrespective of cyclophosphamide (CTX) preconditioning. Lines represent mean; ns: not significant. (C) Comparison of peak responses between patients without CTX preconditioning $(-\mathrm{CTX})$ or receiving CTX course $(+\mathrm{CTX})$ for a week before each immunization, irrespective of vaccination regimen. Lines represent mean; ns: not significant. (D) Time-dependent dynamics of cellular responses to individual 5T4 peptide pools ( $\mathrm{p} 1$ to $\mathrm{p} 8$ ) in 2 representative patients. Arrows represent time points of vaccination. AS, active surveillance; C, ChAdOx1.5T4; M, MVA.5T4; RP, radical prostatectomy.

responses with broad epitope specificities that changed over time (figure 2D).

Twenty-six of $39(67 \%)$ patients also mounted 5T4-specific antibody responses as measured by a relative-quantitative ELISA. ${ }^{27}$ Of note, $5 \mathrm{~T} 4$ seroconversion occurred in all the patients in the standard vaccination regimen arms compared with the $46 \%$ seroconversion rate in patients vaccinated according to the accelerated immunization schedules (online supplementary table S3). There was no correlation between the induced 5T4-specific antibody and T-cell responses (online supplementary figure S2).

In order to evaluate T-cell immune response kinetics, patients on the same vaccination schedule were pooled irrespective of other variables (ie, CTX status pretreatment, and heterologous or homologous vaccination). In patients on the standard schedule, the 5T4-specific T-cell response was observed to peak twice: first at 4 weeks after priming and second 1 week after the second boost (week 9), at a mean of $54 \pm 48$ SEM and $72 \pm 69$ SEM SFCs per million PBMCs, respectively (figure $3 \mathrm{~A}$ ). This observation mirrors the immunogenicity of these vectors in other indications, where responses post-ChAd peak at 2-4 weeks and responses post-MVA peak at 1 week. A peak T-cell response in patients assigned to the accelerated regimen was observed 1 week after the boost at means of $80 \pm 27$ SEM in the surgical arm, and $131 \pm 85$ SEM SFCs per million PBMCs in the AS arm (figure 3B). Following a contraction phase of the immune response, only weak ex vivo responses were detectable at later time points, likely because effector cells diminish while less activated central memory cells increase. ${ }^{15}$ Indeed, the repertoire of memory 5T4-specific $\mathrm{T}$ cells could be expanded by short-term in vitro culture in the presence of the total 5T4 peptide pool from blood samples collected 4-6 months after vaccination. Of note, frequencies of expanded 5T4-specific $\mathrm{T}$ cells from blood samples collected prior to vaccination from the same patients were usually much lower (figure 3D).

Vaccine-induced T-cell responses were further characterized using flow cytometry, which is a less sensitive assay than ELISpot and therefore requires an expansion of the relatively infrequent antigen-specific $\mathrm{T}$ cells. To this end, PBMCs from several patients collected at baseline and at various timepoints during the study were cultured for 12-14 days in presence of the total 5T4 peptide pool as previously mentioned. Following in vitro stimulation, cell cultures were stained with fluorochromelabeled antibodies against $\mathrm{CD} 4+$ and CD8+ T-cell 
A

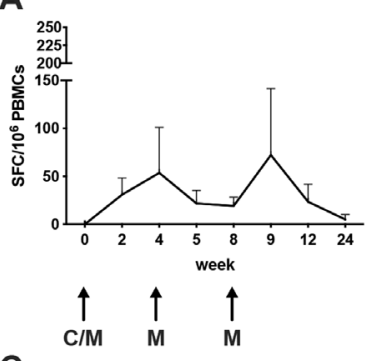

C

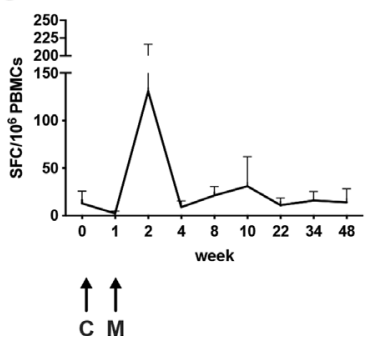

B

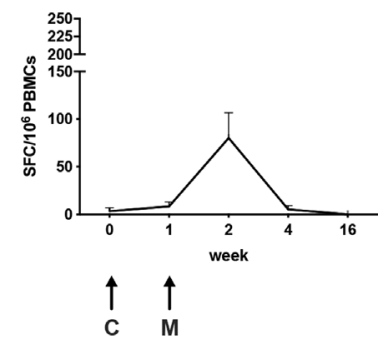

D

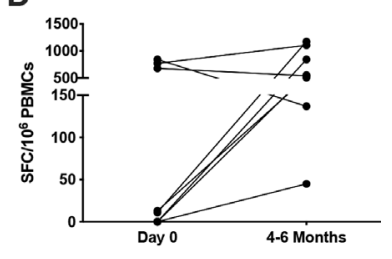

Figure 3 Kinetics of 5T4 specific responses. Antigenspecific responses were measured before vaccination and at different time points post-vaccination by ex vivo IFN- $\gamma$ ELISpot assay on freshly isolated peripheral blood mononuclear cells (PBMCs). Mean $( \pm$ SEM) immune responses to $5 \mathrm{~T} 4$ antigen over time are shown irrespective of cyclophosphamide preconditioning: $(A)$ in the standard vaccination regimen arms; $(B)$ in surgical patients receiving the accelerated vaccination regimen; $(C)$ in active surveillance patients receiving the accelerated vaccination regimen. Arrows represent time points of vaccination. C/M: ChAdOx1.5T4 or MVA.5T4; C: ChAdOx1.5T4; M: MVA.5T4. (D) Freshly isolated PBMCs from several patients were cultured for 2 weeks in the presence of low-dose hlL-2 and the total 5T4 peptide pool. IFN- $\gamma$ ELISpot assay was performed on cultured cells and baseline responses were compared with responses measured at weeks 16 to 24 .

surface markers and intracellular IFN- $\gamma$ and TNF- $\alpha$ cytokines. The gating strategy is presented in figure $4 \mathrm{~A}$ and representative plots of IFN- $\gamma+\mathrm{TNF}-\alpha+\mathrm{CD} 4+$ and CD $8+$ $\mathrm{T}$ cells on 5T4 stimulation are shown in figure 4B. 5T4-specific T-cell responses in cultured PBMCs were detected in 15 of $21(71.4 \%)$ patients analyzed. Among the responders, 5T4-specific CD4+ T-cell reactivity was found in $10(66.7 \%)$ patients, CD8+ T-cell reactivity in $14(93.3 \%)$ patients, and both CD4+ and CD8+ T cellmediated responses in $9(60 \%)$ patients (figure $4 \mathrm{C}$ ). Patients displaying a post-vaccination positive immune response by flow cytometry also had a low percentage of both $\mathrm{CD} 4+$ and $\mathrm{CD} 8+$ polyfunctional $\mathrm{T}$ cells detected at baseline (ranges $0.2 \%-1.8 \%$ and $0.01 \%-0.3 \%$, respectively). However, these numbers at least doubled after vaccination, reaching as high as $5.3 \%$ and $7.8 \%$ of 5T4-specific CD4+ and CD8+ T cells at the peak of the response (figure 4D). The median fold increase of 5T4-specific T-cell responses over baseline values amounted to 4.9 and 4.5 in CD4+ and CD8+ T cells, respectively (figure $4 \mathrm{E}$ ). To corroborate the flow cytometry data on induction of both CD4+ and CD8+ 5T4-specific responses after short-term in vitro stimulation,

PBMCs from selected responders have been retrospectively thawed to perform CD4+ and CD8+ T-cell depletions and to test the depleted cell fractions in parallel with the total PBMCs in an ex vivo IFN- $\gamma$ ELISpot assay (online supplementary materials and methods). As shown in online supplementary table S4, nine patients, including one non-responder, have been analyzed to discriminate between $\mathrm{CD} 4+$ and $\mathrm{CD} 8+\mathrm{T}$-cell responses ex vivo. Among the responders, only four maintained the response after freeze/thawing and we could observe CD4+ only mediated, CD8+ only mediated, and both CD4+ and CD8+ mediated T-cell responses, consistent with the results observed by flow cytometry.

\section{Vaccine-induced T-cell responses in the prostate}

In order to assess the immune response to the vaccine antigen in the target prostate gland, post-vaccination tissue cores from RP specimens ${ }^{28}$ and prostate biopsy samples from AS patients were cultured in vitro in order to expand TILs and enrich them for 5T4 specificity prior to flow cytometric analysis. Immune cell expansion rates and $\mathrm{CD} 4+$ to $\mathrm{CD} 8+\mathrm{T}$-cell ratios varied between patients, as well as between tissue fragments derived from the same surgical or biopsy specimen. Representative dot plots of expanded CD4+ and CD8+ T cells are shown in figure 5A. The flow cytometric analysis of TILs was similar to that performed on cultured PBMCs. 5T4-specific T-cell responses could be detected in the prostate samples of 13 of 17 patients analyzed ( $76.5 \%$ response rate). Overall, prostate tissue analysis demonstrated the presence of 5T4-specific CD4+ T-cell reactivity in 8 patients $(61.5 \%$ of responders), CD8+ T-cell reactivity in 11 patients $(84.6 \%$ of responders) and both CD4+ and CD8+ T cell-mediated responses in 6 patients ( $46.2 \%$ of responders) (figure $5 \mathrm{~B}$ ). Percentages of TILs secreting both IFN- $\gamma$ andTNF- $\alpha$ measured in two representative patients are shown as a comparison between unstimulated and 5T4-stimulated cells in figure $5 \mathrm{C}$.

To evaluate post-treatment changes in the immune cell infiltration using immunohistochemistry (IHC), tissue sections from AS patients were stained with anti-CD3 and anti-CD8 monoclonal antibody, and densities of CD3+ and $\mathrm{CD} 8+\mathrm{T}$ cells were assessed by digital image analysis as previously described ${ }^{29}$ An increase in CD3+ and CD8+ T-cell density was observed in the post-vaccination prostate gland in some of the analyzed patients. In particular, $\mathrm{a} \geq 1$.5-fold increase in CD3+ and CD8+ T-cell density posttreatment was detected in 2 out of 11 patients $(18 \%)$ and in 4 out of 11 patients (36\%), respectively (online supplementary figure S3).

IHC analysis was also performed on 12 available RP tissue sections from patients in groups $1-4$, and both CD3+ and CD8+ T-cell infiltration was detected in resection samples (online supplementary table S5). However, T-cell densities in the prostate gland following vaccinations could not be reliably compared with the pretreatment archival biopsy samples due to technical issues. 
A
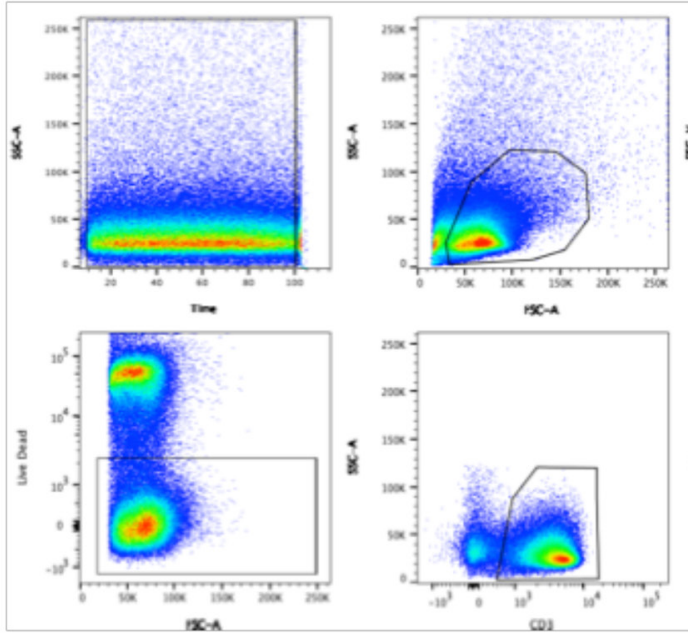

D

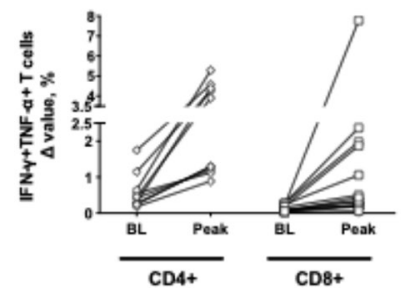

B
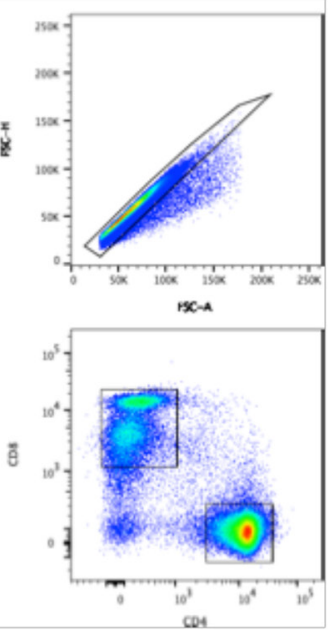

E

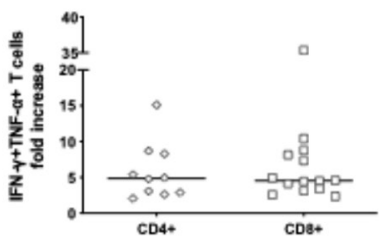

Figure 4 Flow cytometry analysis of 5T4-specific immune responses induced by vaccination. Freshly isolated peripheral blood mononuclear cells (PBMCs) were cultured for 2 weeks in the presence of low-dose hIL-2 and the total 5T4 peptide pool to expand relatively infrequent 5T4-specific T cells. Flow cytometric analysis was performed on cultured cells at different time points, and the percentage of polyfunctional CD4+ and CD $8+$ secreting IFN- $\gamma+$ and TNF- $\alpha+$ was calculated by subtracting background responses ( $\Delta$ value) and compared with corresponding pre-vaccination values. (A) Representative gating strategy. From left to right, top to bottom: time-lymphocytes-single cells-live cells-CD3+ T cells-CD4+ and CD8+ T cells. (B) Representative dot plot with percentages showing CD4+ (top) and CD8+ (bottom) T cells secreting IFN- $\gamma+$ and TNF- $\alpha+$. (C) Percentages of evaluated patients showing a positive response in cultured PBMCs (gray bar) and relative percentages of positivity for CD4+, CD8+, and both CD4+ and CD8+ among responders (black bars). (D) Baseline $\Delta$ value percentages of IFN- $\gamma+$ and TNF- $\alpha+C D 4+$ and CD8+ T cells are compared with corresponding peak $\Delta$ value percentages obtained after vaccination in each individual patient analyzed. BL: baseline. (E) Fold increase of peak $\Delta$ value percentages over baseline of polyfunctional CD4+ and CD8+ T cells induced by vaccination are shown. Lines represent median.

\section{Serum PSA changes following vaccination}

The serum PSA level is commonly used as a surrogate marker of PCa treatment efficacy. Potential treatment effects of the vaccine on PCa were assessed by measuring serum PSA throughout the trial. In the majority of patients in the surgical arms, PSA could only be assessed at a few time points prior to RP, as this intervention ordinarily causes the PSA to drop to very low or undetectable levels. However, patients on the AS program could be followed up for 48 weeks, thus changes in PSA were monitored in these patients for an extended time period. Figure 6A shows PSA changes over time in the 12 patients in the AS arms. Although there was some disparity in the PSA kinetics between patients, the overall trend showed an unexpected increase in serum PSA concentration after ChAdOx1.5T4 prime with a peak at week 4, with PSA decreasing to pre-vaccination levels by week 22 (figure 6B). Of note, median peak levels of PSA after vaccination were significantly higher than median baseline levels $(8.25 \mathrm{ng} / \mathrm{mL}$ vs $4.16 \mathrm{ng} / \mathrm{mL}$ ) (figure $6 \mathrm{C}$ ).
Analysis of the maximal PSA change detected at any given time point post-vaccination demonstrated that $25 \%$ of the patients had a PSA drop of 12\%-38\% compared with pre-vaccination values and $50 \%$ of patients experienced an increase of $17 \%-52 \%$ over the baseline (figure 6D). Notably, $25 \%$ of patients had a striking $\geq 100 \%$ increase in PSA concentration from a baseline mean of $3.9 \pm 0.5 \mathrm{SD} \mathrm{ng} / \mathrm{mL}$ to a peak mean of $10.7 \pm 1.4 \mathrm{SD}$ $\mathrm{ng} / \mathrm{mL}$ (figure 6D). Importantly, this increase of $\geq 100 \%$ in serum PSA levels observed in three patients was associated with both peripheral and local biomarkers of vaccine immunological activity, such as presence of polyfunctional 5T4-specific CD8+ and CD4+ T-cell responses in the blood as measured by ex vivo ELISpot and flow cytometry of short-term cultured PBMCs, expansion of 5T4-specific polyfunctional $\mathrm{T}$ cells from fresh prostate biopsy samples and a $\geq 1$.5-fold increase in CD8+ T-cell infiltration in post-treatment prostate biopsies compared with baseline (table 1). T-cell densities measured by IHC of two of these three patients are shown in figure 7 , as well 
A

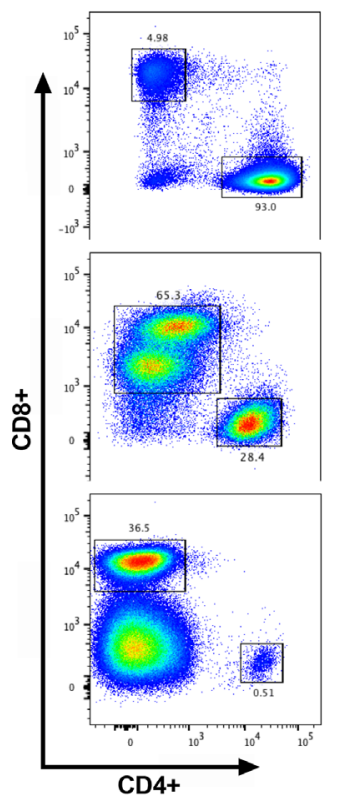

B

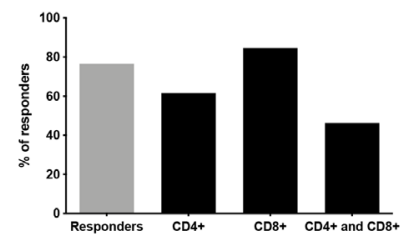

C
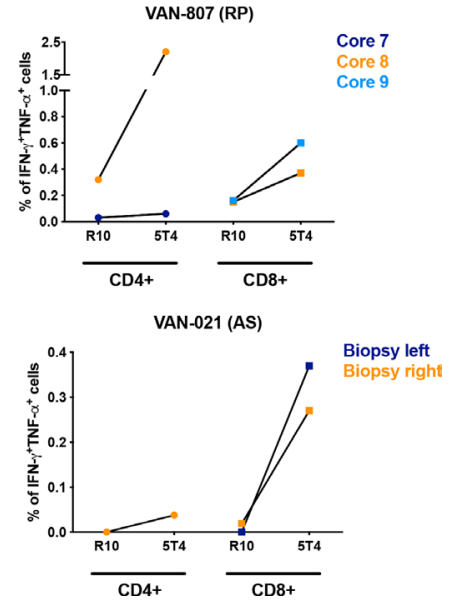

Figure 5 Flow cytometry analysis of 5T4 specific immune responses in the prostate tissue. Post-vaccination prostate biopsy samples and radical prostatectomy tissue specimens were cultured in the presence of high-dose hIL-2 and were stimulated with the total 5T4 peptide pool to expand 5T4-specific $T$ cells derived from the prostate gland. Flow cytometric analysis was performed on expanded tumorinfiltrating lymphocytes (TILs) 1 week after 5T4 stimulation. (A) Representative flow dot plot showing percentages of gated CD4+ and CD8+ TILs obtained from expanded cultures in different samples. (B) Percentages of evaluated samples showing a positive response in cultured TILs (gray bar) and relative percentages of positivity for the CD4+, CD8+ Tcell and both CD4+ and CD8+ T-cell subsets (black bars). (C) Percentages of IFN- $\gamma+$ and TNF- $\alpha+$ TILs (CD4+ and CD8+) in unstimulated cultures (R10: medium only) are compared with the corresponding 5T4-stimulated cultures (5T4) in 2 representative patients (VAN-807 and VAN-021). AS, active surveillance; RP, radical prostatectomy.

as representative images of IHC staining of the respective prostate biopsies. The TIL and IHC data for the third patient (VAN-022) were not available as his on-study biopsy was not performed for clinical reasons.

\section{DISCUSSION}

In this study, the safety profile and immunogenicity of the first-in-man ChAdOx1-MVA vaccination against the 5T4 tumor antigen in low-risk and intermediate-risk patients with PCa has been evaluated. The vaccine was well tolerated in both the standard and accelerated regimens, with the safety profile in agreement with previous reports for these viral vectors. ${ }^{13} 3031$ Vaccines induced de novo 5T4-specific immunity in all but two patients and significantly boosted two pre-existing responses. Overall,
$64 \%$ of patients were found to have responded to vaccination with a magnitude of response ranging between 30 and 1025 SFCs per million PBMCs and some responses detected as early as after a single priming immunization. To our knowledge, this is the best vaccine-induced immune response rate obtained to date in any clinical trials in patients with $\mathrm{PCa}$ measured by ex vivo IFN- $\gamma$ ELISpot assay.

Vaccination-induced 5T4-specific antibody responses were detected in all patients in the standard vaccination regimen arms, as opposed to $46 \%$ of patients following the accelerated vaccination protocol. This finding suggests that a second MVA boost and/or longer intervals between vaccinations are more favorable for induction of antibody responses to 5T4.

Available information on breaking tolerance and induction of T-cell responses to self-antigens in PCa vaccine trials remains sparse. The response rate and magnitude of response achieved in the VANCE trial can be considered noteworthy when compared with immunotherapy responses measured in other clinical trials on patients with PCa. However, to date the majority of PCa immunotherapy clinical trials have been focused on patients with metastatic castration-resistant disease. These patients are known to have compromised cellular immunity, increased suppressive phenotypes of myeloidderived suppressor cells and regulatory $\mathrm{T}$ cells in the circulation, and an increased immune suppressive tumor microenvironment. These factors have to be taken into account when comparing the immune responses elicited in the patients with low-risk/intermediate-risk low-volume disease recruited to the VANCE study.

The closest comparator for the ChAdOx1-MVA 5T4 vaccine in the metastatic PCa setting is TroVax, a homologous MVA 5T4 vaccination regimen. ${ }^{30}$ In the reported phase II trial, 5T4-specific T-cell responses were induced in $38 \%$ of patients with hormone refractory PCa over the course of 11 TroVax immunizations. ProstVac vaccine induced PSA-specific T-cell responses in 57\% of patients across six clinical trials. However, the magnitude of the response measured 4 weeks after the last immunization was in the range of 10 to 203 SFU per million PBMCs, with a median response of $30 \mathrm{SFU}$ per million PBMCs. ${ }^{4}$ Recently, ProstVac vaccine has been evaluated in the neoadjuvant setting prior to radical prostatectomy in a patient cohort similar to the one in the VANCE study. ${ }^{32} \mathrm{~A}$ direct comparison of the immune responses induced by ProstVac and ChAd-MVA vaccines is difficult because of the different primary outcome measures and test assays applied. Nevertheless, ProstVac vaccination has led to increased CD4+ T-cell infiltration in the RP specimens compared with pre-treatment biopsies in more than onethird of the patients, and $28 \%$ of the patients developed PSA-specific CD4+ and CD8+ T-cell responses as measured by flow cytometry following a period of in vitro stimulation with overlapping PSA peptides.

The licensed cell-based immunotherapy Sipuleucel-T induced T-cell responses to the target antigen, 
A
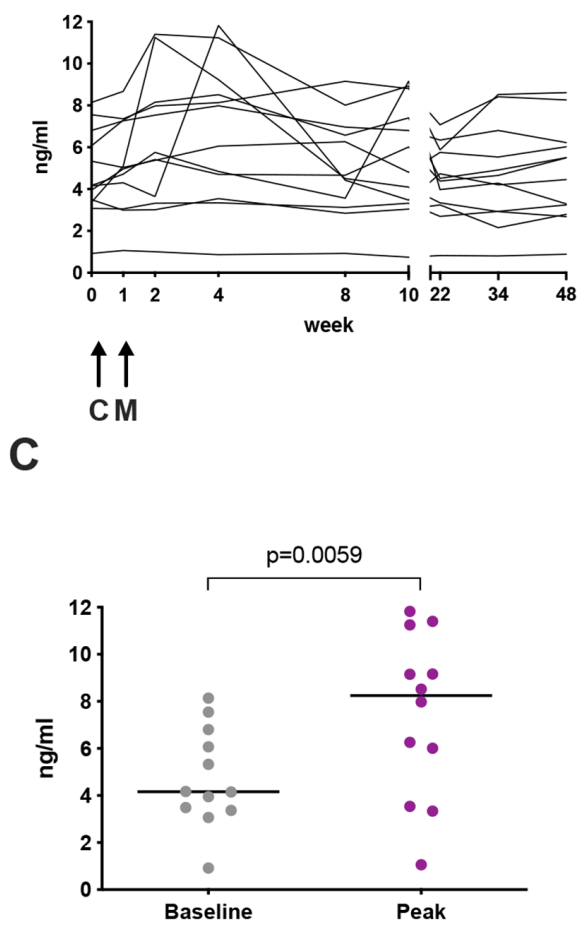

B

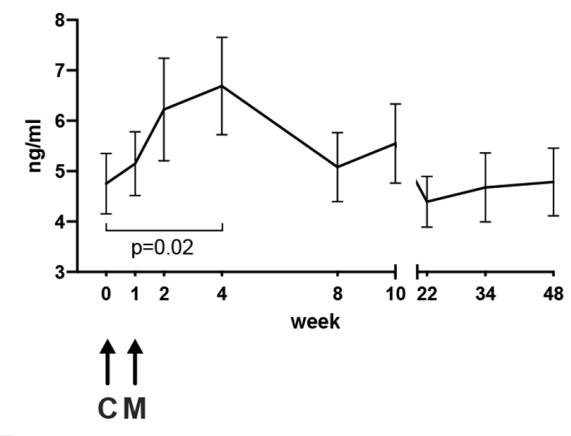

D

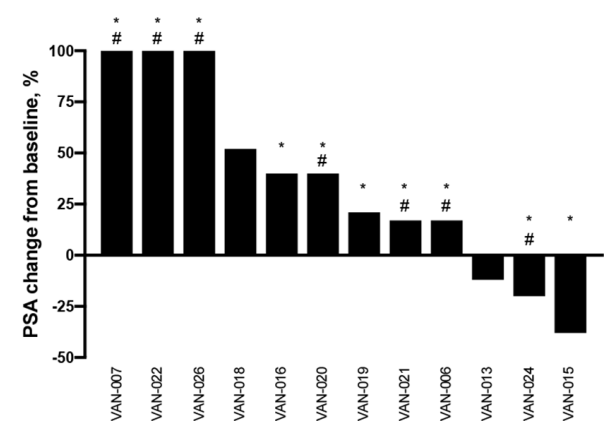

Figure 6 Prostate-specific antigen (PSA) levels in the active surveillance arms. Serum PSA concentration was measured throughout the study. Changes in PSA concentration are shown for each individual patient in the active surveillance arms $(A)$ and as mean concentrations \pm SEM (B); paired t-test value $p=0.02$. Arrows represent time points of vaccination. C: ChAdOx1.5T4; M: MVA.5T4. (C) A scatter dot plot graph representing baseline PSA concentrations compared with corresponding peak concentrations measured post-vaccination in active surveillance patients. Lines represent median; paired t-test value $p=0.006$. (D) Waterfall plot representing the highest PSA change from baseline, expressed as percentage of baseline value, measured at any given timepoint in patients on the active surveillance arms (patient trial IDs are given). ${ }^{*}$ represents a systemic immune response to the vaccine measured either by ex vivo IFN- $\gamma$ ELISpot on freshly isolated peripheral blood mononuclear cells (PBMCs) or flow cytometry on short-term stimulated PBMCs. \# indicates a CD8+ T-cell local immune response to the vaccine measured either by flow cytometry on short-term stimulated tumor-infiltrating lymphocyte cultures or by immunohistochemistry on post-vaccination biopsies (CD8+ T-cell density $\geq 1.5$-fold pre-vaccination biopsies).

a recombinant fusion protein of PAP and GM-CSF (PA2024), in $48 \%$ of patients with treated mCRPC compared with $6 \%$ in the control group. However, the response rate to the PAP antigen itself was in the range of $6 \%-8 \%$ in both treated and control patients, ${ }^{3}$ which suggests that much of T-cell reactivity against the recombinant protein may have been directed to the junctional foreign antigens. In another clinical trial, a DNA vaccine encoding PAP and GM-CSF (pTVG-HP) was assessed for immunogenicity in patients with PCa with biochemical recurrence. This vaccine yielded a low response rate of $14 \% .{ }^{33} \mathrm{~A}$ follow-up study demonstrated that PAP-specific $\mathrm{T}$ cells could be detected after a course of six vaccinations. ${ }^{34}$ The pTVG-HP vaccine has been evaluated more recently in mCRPC in combination with anti-PD $1 .^{35}$ The reported T-cell responses were likely to be predominantly CD4+ T cell mediated, as the PAP protein induced much higher frequencies of PAP-specific $\mathrm{T}$ cells by IFN- $\gamma$ ELISpot compared with the PAP peptide antigen. Also, the responses to PAP peptides were detected after $48-72$ hours of in vitro re-stimulation, in contrast to the standard 18-hour assay used here. An RNA-based vaccination approach has also been evaluated in mCRPC. ${ }^{36}$ The self-adjuvanted mRNA vaccine CV1903 against four prostate antigens induced T-cell responses in 55\% of patients. These responses were detected after six doses of the vaccine and reported as a cumulative response rate measured by MHC class I tetramer staining, ICS and IFN- $\gamma$ ELISpot.

CTX preconditioning did not have a detectable effect on the magnitude of 5T4-specific response. This observation was unexpected, as low-dose CTX may selectively suppress regulatory $\mathrm{T}$ cell (Treg) number and functionality, without influencing other T-cell subsets. ${ }^{37}$ However, the little impact from CTX observed here is corroborated by other studies. For example, depletion of Tregs with low-dose CTX prior to vaccination did not increase immune responses generated by MVA.5T4 vaccination in patients with metastatic colorectal cancer. ${ }^{38}$

We observed the presence of vaccine-induced systemic CD8+ or/and CD4+ T-cell responses and also detected CD8+ T-cell infiltration into the prostate by IHC. 


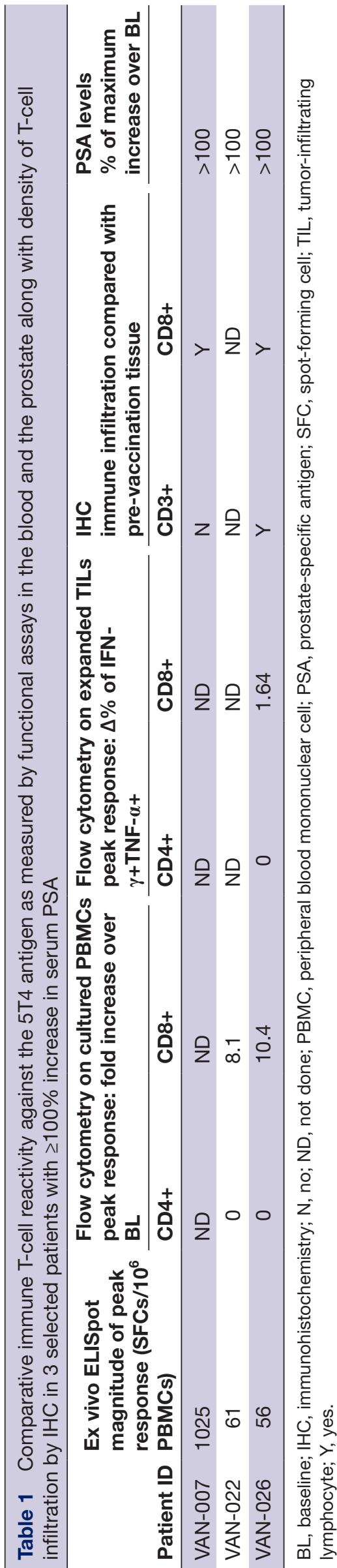

Interestingly, in contrast to observations seen in other malignancies, a positive correlation between the density of tumor-infiltrating CD8+ T cells and improved clinical outcome remains controversial in $\mathrm{PCa}^{39}$ On one hand, it has been suggested that abundant TILs in the prostate gland are associated with a higher survival probability. ${ }^{40}$ On the other hand, high numbers of TILs within the tumor have been predictive of an increased risk of tumor recurrence in patients undergoing $\mathrm{RP} .{ }^{41}{ }^{42}$ In addition, a strong TIL presence was an independent predictor of short PSA recurrence-free survival. ${ }^{43}$ More recently, higher levels of primarily CD4+ T cells and, to a lesser extent, CD8+ T cells, appeared to be associated with a worse distant metastasis-free survival. ${ }^{44}$ These reports suggest that in addition to enumerating $\mathrm{T}$ cells in the prostate tumor, the functional status and tumor specificity of TILs after vaccination are important characteristics to analyze. To this end, in the VANCE study, we made use of fresh post-vaccination prostate tissue for quantitative assessment of 5T4-specific polyfunctional TILs in addition to IHC analysis of fixed tissue. Of note, 11 of $13(85 \%)$ patients demonstrated presence of functional CD8+ T cells, with over $50 \%$ of these patients exhibiting a concomitant CD4+ T-cell response. A limitation of our approach is that the TILs subjected to this functional analysis have been cultured in vitro, isolated from the natural tumor immunosuppressive microenvironment, and so they might have expanded in a more favorable environment. Another drawback of our analysis is that it was not possible to compare the immune cell phenotype and function in the prostate gland pre-vaccination and post- vaccination, due to the unavailability of fresh tissue before study treatment.

The presence of 5T4-reactive TILs might also explain the transient increase in PSA levels observed in AS patients post-vaccination. Although the observed PSA trends post-vaccination were variable, three AS patients $(25 \%)$ experienced a transient PSA rise of $\geq 100 \%$ that cannot be explained by physiological variations or inter-test variability. The herein hypothesized correlation between PSA rise and the local vaccine-specific T-cell reactivity remains to be investigated further. However, to corroborate our speculation, a "PSA bounce" observed after brachytherapy in localized PCa correlated with higher densities of CD3+ and $\mathrm{CD} 8+\mathrm{T}$ cells within the tumor, suggesting that local immune response was responsible for the PSA bounce observed in the analyzed cohort of patients with early $\mathrm{PCa}^{45}$

\section{CONCLUSIONS}

The heterologous ChAdOx1-MVA 5T4 vaccination regimen in early-stage $\mathrm{PCa}$ was found to be both safe and immunogenic, with the majority of patients with PCa enrolled in this trial mounting ex vivo T-cell responses to the vaccine-encoded tumor-associated antigen 5T4. Immune responses mediated by polyfunctional CD8+ and $\mathrm{CD} 4+\mathrm{T}$ cells could be detected following vaccination 
A
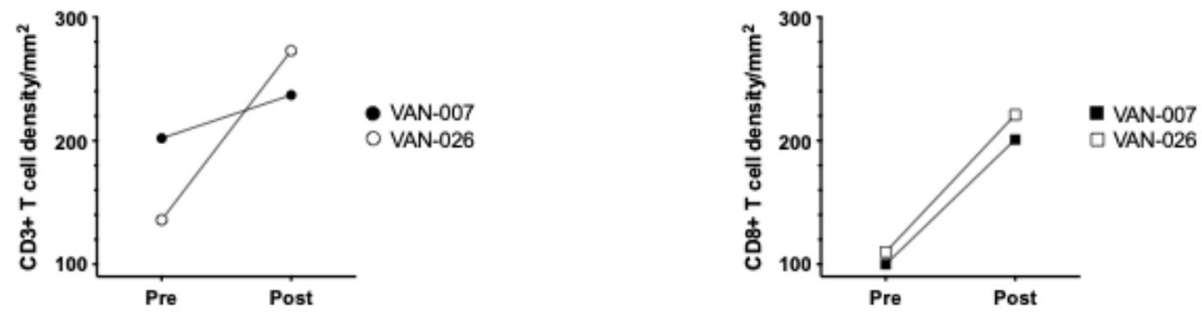

B
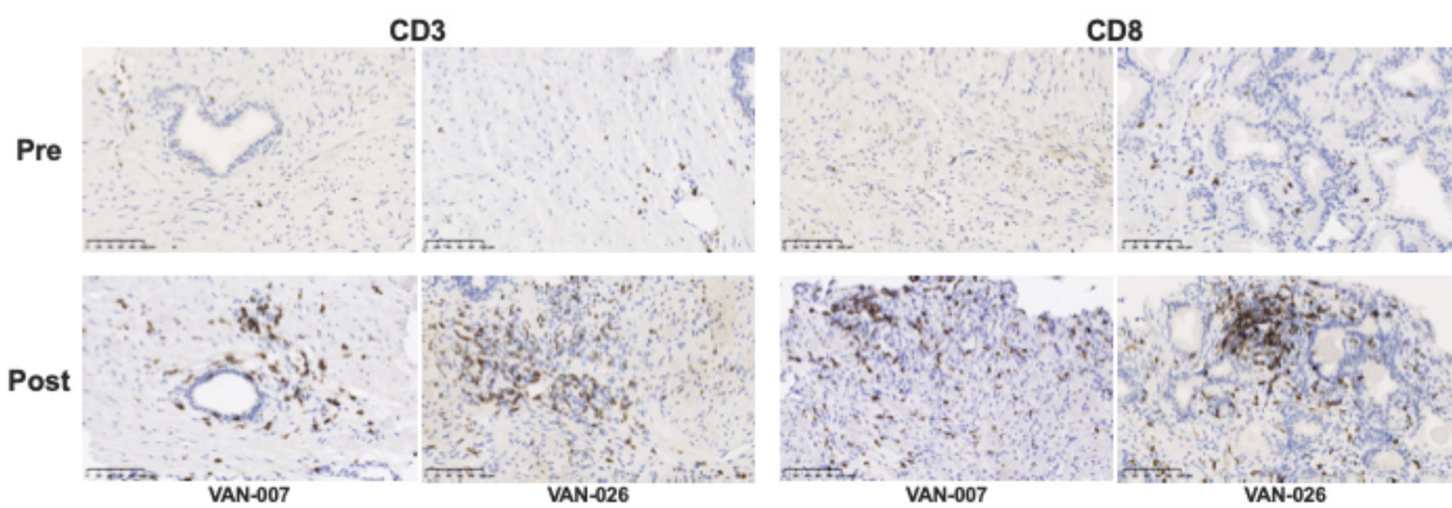

Figure 7 T-cell infiltration in the prostate. Archival diagnostic prostate biopsies and matched on-study prostate biopsies taken at week 10 from selected patients in the active surveillance arms were tested for T-cell infiltration by immunohistochemistry (patient trial IDs are given). Formalin-fixed paraffin-embedded sections were stained with anti-CD3 and anti-CD8 antibodies and cell densities were calculated. (A) Comparison of CD3+ (left) and CD8+ (right) T-cell densities, expressed as number of cells $/ \mathrm{mm}^{2}$, and (B) representative pictures of CD3 and CD8 expression between pre-treatment and post-treatment biopsies in 2 patients who had $\geq 100 \%$ increase in serum prostate-specific antigen levels post-vaccination. Brown regions indicate immunoreactivity. Scale bar is shown $(100 \mu \mathrm{m})$.

ex vivo in the blood and following in vitro culture of cells obtained from prostate tissue. The transient rise in serum PSA observed post-vaccination, which significantly exceeded physiological variation in some patients, supports a possible vaccination effect within the prostate gland.

It remains to be seen if the vaccination strategy presented herein generates immune responses of sufficient magnitude to mediate clinical efficacy and whether it can be effective in later-stage PCa therapeutic settings, as a monotherapy in advanced disease or as part of multi-modality PCa therapy. To address these questions, we have started the phase I/II trial ADVANCE, which is currently recruiting patients with intermediate-risk $\mathrm{PCa}$, and patients with advanced mCRPC, to receive ChAdOx1-MVA 5T4 vaccine in combination with nivolumab (NCT03815942).

\section{Author affiliations}

${ }^{1}$ Nuffield Department of Medicine, The Jenner Institute, Oxford University, Oxford, UK

${ }^{2}$ Nuffield Department of Surgical Sciences, 0xford University, Oxford, UK

${ }^{3}$ Department of Urology, Churchill Hospital, Oxford, UK

${ }^{4}$ Oxford NIHR Biomedical Research Centre, 0xford University, 0xford, UK

${ }^{5}$ Definiens AG, Munchen, Bayern, Germany

${ }^{6}$ Oxford Biomedica PIc, Oxford, UK
${ }^{7}$ Department of Oncology, Oxford Cancer and Haematology Centre, Churchill Hospital, Oxford, UK

${ }^{8}$ Department of Radiology, Churchill Hospital, Oxford, UK

${ }^{9}$ Department of Radiology, Sheffield Teaching Hospitals NHS Foundation Trust, Sheffield, UK

${ }^{10}$ Department of Pathology, Sheffield Teaching Hospitals NHS Foundation Trust, Sheffield, UK

${ }^{11}$ Oncology, University Hospital of Lausanne, Lausanne, Switzerland

${ }^{12}$ Vaccitech UK Limited, Oxford, UK

${ }^{13}$ Academic Urology Unit, The University of Sheffield, Sheffield, UK

Twitter Pedro J Romero @JITCancer

Acknowledgements We thank the trial participants and their families for their involvement in this study, the study teams at the local sites for assistance in the execution of this clinical trial and the Oxford Centre for Histopathology Research for processing samples for IHC. We acknowledge support of Clare Verrill by the NIHR Oxford Biomedical Research Centre. We are grateful to Oxford Biomedica Ltd for supplying the MVA.5T4 vaccine.

Contributors Trial conception and design: FH, AVSH, IR. Experimental design, data analysis and interpretation: FC, AVSH, IR. Laboratory data generation: FC, EP, AM, GS, RH, SV. Provision of patients: RB, LC, JH, JC, FH. Collection of biological samples or clinical data: CV, IP, MB, CM, AP, RMP, SK, SM. Protocol development: RB, LC, PJR, TE, FH, AVSH, IR. Administrative support: AB. Manuscript writing: FC, IR. Manuscript review: RB, CV, AM, GS, RH, SV, PJR, TE, JC, FH, AVSH. Final approval of manuscript: all authors. AVSH and IR contributed equally to this work.

Funding The VANCE clinical trial was supported by the European Union's Seventh Framework Programme under grant agreement no. 602705. 
Competing interests AVSH is a co-founder of and shareholder in Vaccitech Ltd which has supported the 0xford prostate cancer vaccine programme.

\section{Patient consent for publication Not required.}

Ethics approval This study was approved within the UK by the Medicines and Healthcare Products Regulatory Agency (Ref. 21584/0338/001-0001) and the South Central Berkshire Research Ethics Committee (Ref. 14/SC/1231). Written informed consent was obtained from all participating patients, and the trial was conducted in accordance with the principles of the Declaration of Helsinki and Good Clinical Practice.

\section{Provenance and peer review Not commissioned; externally peer reviewed.}

Data availability statement The datasets used and/or analyzed during the current study are available from the corresponding author on reasonable request.

Open access This is an open access article distributed in accordance with the Creative Commons Attribution Non Commercial (CC BY-NC 4.0) license, which permits others to distribute, remix, adapt, build upon this work non-commercially, and license their derivative works on different terms, provided the original work is properly cited, appropriate credit is given, any changes made indicated, and the use is non-commercial. See http://creativecommons.org/licenses/by-nc/4.0/.

\section{ORCID iD}

Irina Redchenko http://orcid.org/0000-0002-3234-3548

\section{REFERENCES}

1 Culp MB, Soerjomataram I, Efstathiou JA, et al. Recent global patterns in prostate cancer incidence and mortality rates. Eur Urol 2020;77:38-52.

2 Kantoff PW, Higano CS, Shore ND, et al. Sipuleucel-T immunotherapy for castration-resistant prostate cancer. $N$ Engl $J$ Med 2010;363:411-22.

3 Sheikh NAet al. Sipuleucel-T immune parameters correlate with survival: an analysis of the randomized phase 3 clinical trials in men with castration-resistant prostate cancer. Cancer immunology, immunotherapy: CII, 2013: 62. 137-47.

4 Gulley JLet al. Immune impact induced by PROSTVAC (PSATRICOM), a therapeutic vaccine for prostate cancer. Cancer Immunology Research 2013;2:133141.

5 Gulley JL, Borre M, Vogelzang NJ, et al. Phase III trial of PROSTVAC in asymptomatic or minimally symptomatic metastatic castrationresistant prostate cancer. J Clin Oncol 2019;37:1051-61.

6 Hole N, Stern PL. Isolation and characterization of 5T4, a tumourassociated antigen. Int J Cancer 1990;45:179-84.

7 Southall PJ, Boxer GM, Bagshawe KD, et al. Immunohistological distribution of 5T4 antigen in normal and malignant tissues. $\mathrm{Br} J$ Cancer 1990;61:89-95.

8 Starzynska T, Rahi V, Stern PL. The expression of 5T4 antigen in colorectal and gastric carcinoma. Br J Cancer 1992;66:867-9.

9 Wrigley E, McGown AT, Rennison J, et al. 5T4 oncofetal antigen expression in ovarian carcinoma. Int $J$ Gynecol Cancer 1995;5:269-74.

10 Griffiths RW, Gilham DE, Dangoor A, et al. Expression of the 5T4 oncofoetal antigen in renal cell carcinoma: a potential target for Tcell-based immunotherapy. Br J Cancer 2005;93:670-7.

11 Al-Taei S, Salimu J, Lester JF, et al. Overexpression and potential targeting of the oncofoetal antigen 5T4 in malignant pleural mesothelioma. Lung Cancer 2012;77:312-8.

12 Ewer KJ, O'Hara GA, Duncan CJA, et al. Protective CD8+ T-cell immunity to human malaria induced by chimpanzee adenovirus-MVA immunisation. Nat Commun 2013;4:2836.

13 Antrobus RD, Coughlan L, Berthoud TK, et al. Clinical assessment of a novel recombinant simian adenovirus ChAdOX1 as a vectored vaccine expressing conserved influenza $\mathrm{A}$ antigens. Mol Ther 2014;22:668-74.

14 Borthwick N, Ahmed T, Ondondo B, et al. Vaccine-elicited human T cells recognizing conserved protein regions inhibit HIV-1. Mol Ther 2014;22:464-75.

15 Swadling L, Capone S, Antrobus RD, et al. A human vaccine strategy based on chimpanzee adenoviral and MVA vectors that primes, boosts, and sustains functional HCV-specific T cell memory. Sci Transl Med 2014;6:ra153.

16 Ewer K, Rampling T, Venkatraman N, et al. A monovalent chimpanzee adenovirus Ebola vaccine boosted with MVA. N Engl J Med 2016;374:1635-46.

17 Tiono $A B$, Nébié I, Anagnostou N, et al. First field efficacy trial of the ChAd63 MVA ME-TRAP vectored malaria vaccine candidate in 5-17 months old infants and children. PLoS One 2018;13:e0208328.
18 Venkatraman N, Ndiaye BP, Bowyer G, et al. Safety and immunogenicity of a heterologous prime-boost Ebola virus vaccine regimen in healthy adults in the United Kingdom and Senegal. $J$ Infect Dis 2019;219:1187-97.

19 Cappuccini F, Stribbling S, Pollock E, et al. Immunogenicity and efficacy of the novel cancer vaccine based on simian adenovirus and MVA vectors alone and in combination with PD-1 mAb in a mouse model of prostate cancer. Cancer Immunology, Immunotherapy 2016;65:701-13.

20 Cappuccini F, Pollock E, Stribbling S, et al. 5T4 oncofoetal glycoprotein: an old target for a novel prostate cancer immunotherapy. Oncotarget 2017;8:47474-89.

21 Kim DW, Krishnamurthy V, Bines SD, et al. TroVax, a recombinant modified vaccinia Ankara virus encoding 5T4: lessons learned and future development. Hum Vaccin 2010;6:784-91.

22 Nguyen LT, Yen PH, Nie J, et al. Expansion and characterization of human melanoma tumor-infiltrating lymphocytes (TILs). PLoS One 2010;5:e13940.

23 Brieu N, Pauly O, Zimmermann J, et al. Slide-specific models for segmentation of differently stained digital histopathology whole slide images 2016:9784.

24 Kapil A, Meier A, Zuraw A, et al. Deep semi supervised generative learning for automated tumor proportion scoring on NSCLC tissue needle biopsies. Sci Rep 2018;8:17343.

25 McConkey SJ, Reece WHH, Moorthy VS, et al. Enhanced Tcell immunogenicity of plasmid DNA vaccines boosted by recombinant modified vaccinia virus Ankara in humans. Nat Med 2003;9:729-35.

26 Afolabi MO, Ndure J, Drammeh A, et al. A phase I randomized clinical trial of candidate human immunodeficiency virus type 1 vaccine MVA.HIVA administered to Gambian infants. PLoS One 2013;8:e78289.

27 Harrop Ret al. MVA-5T4-induced immune responses are an early marker of efficacy in renal cancer patients. Cancer immunology, immunotherapy: CII, 2011: 60. 829-37.

28 Gill PS, Roberts ISD, Browning L, et al. The handling and sampling of radical prostatectomy specimens for reporting and research: the Oxford approach. J Clin Pathol 2012;65:1057-61.

29 Harder N, Athelogou M, Hessel H, et al. Tissue phenomics for prognostic biomarker discovery in low- and intermediate-risk prostate cancer. Sci Rep 2018;8:4470.

30 Amato RJ, Drury N, Naylor S, et al. Vaccination of prostate cancer patients with modified vaccinia Ankara delivering the tumor antigen 5T4 (TroVax): a phase 2 trial. J Immunother 2008;31:577-85.

31 Coughlan L, Sridhar S, Payne R, et al. Heterologous two-dose vaccination with simian adenovirus and poxvirus vectors elicits long-lasting cellular immunity to influenza virus $A$ in healthy adults. EBioMedicine 2018;29:146-54.

32 Abdul Sater H, Marté JL, Donahue RN, et al. Neoadjuvant PROSTVAC prior to radical prostatectomy enhances T-cell infiltration into the tumor immune microenvironment in men with prostate cancer. J Immunother Cancer 2020;8:e000655.

33 McNeel DG, Dunphy EJ, Davies JG, et al. Safety and immunological efficacy of a DNA vaccine encoding prostatic acid phosphatase in patients with stage D0 prostate cancer. J Clin Oncol 2009;27:4047-54.

34 Becker JT, Olson BM, Johnson LE, et al. DNA vaccine encoding prostatic acid phosphatase (PAP) elicits long-term T-cell responses in patients with recurrent prostate cancer. $J$ Immunother 2010;33:639-47.

35 McNeel DG, Eickhoff JC, Wargowski E, et al. Concurrent, but not sequential, PD-1 blockade with a DNA vaccine elicits anti-tumor responses in patients with metastatic, castration-resistant prostate cancer. Oncotarget 2018;9:25586-96.

36 Kübler H, Scheel B, Gnad-Vogt U, et al. Self-adjuvanted mRNA vaccination in advanced prostate cancer patients: a first-in-man phase I/Ila study. J Immunother Cancer 2015;3:26.

37 Le DT, Jaffee EM. Regulatory T-cell modulation using cyclophosphamide in vaccine approaches: a current perspective. Cancer Res 2012;72:3439-44.

38 Scurr Met al. Effect of modified vaccinia Ankara-5T4 and low-dose cyclophosphamide on antitumor immunity in metastatic colorectal cancer: A randomized clinical trial. JAMA Oncology 2017.

39 Strasner A, Karin M. Immune infiltration and prostate cancer. Front Oncol 2015;5:128.

40 Vesalainen S, Lipponen P, Talja M, et al. Histological grade, perineural infiltration, tumour-infiltrating lymphocytes and apoptosis as determinants of long-term prognosis in prostatic adenocarcinoma. Eur J Cancer 1994;30A:1797-803.

41 Irani J, Goujon JM, Ragni E, et al. High-Grade inflammation in prostate cancer as a prognostic factor for biochemical recurrence 
after radical prostatectomy. pathologist multi center Study Group. Urology 1999;54:467-72.

42 McArdle PA, Canna K, McMillan DC, et al. The relationship between T-lymphocyte subset infiltration and survival in patients with prostate cancer. Br J Cancer 2004;91:541-3.

43 Kärjä V, Aaltomaa S, Lipponen P, et al. Tumour-Infiltrating lymphocytes: a prognostic factor of PSA-free survival in patients with local prostate carcinoma treated by radical prostatectomy. Anticancer Res 2005;25:4435-8.

44 Zhao SG, Lehrer J, Chang SL, et al. The immune landscape of prostate cancer and nomination of PD-L2 as a potential therapeutic target. J Natl Cancer Inst 2019;111:301-10.

45 Yamamoto Y, Offord CP, Kimura G, et al. Tumour and immune cell dynamics explain the PSA bounce after prostate cancer brachytherapy. Br J Cancer 2016;115:195-202. 